\title{
Improvements and testing practical expressions for photon strength functions of E1 gamma-transitions
}

\author{
Vladimir Plujko a , Oleksandr Gorbachenko, Igor Kadenko, and Kateryna Solodovnyk \\ Nuclear Physics Department, Taras Shevchenko National University, Kyiv, Ukraine
}

\begin{abstract}
Analytical expression for the E1 photon strength functions (PSF) is modified to account for the low-energy enhancement due to nuclear structure effects (presence of pygmy dipole resonance (PDR)). A closed-form expression of the E1 PSF function includes response of two nuclear states - PDR and giant dipole resonance (GDR). Expression for the nuclear response function on electromagnetic field is based on a model of excitation of two coupled damped states. This approach is tested for different data sets for spherical nuclei. Impact on the PSF shape of coupling between the PDR and GDR excitations is considered.
\end{abstract}

\section{Introduction}

The average probabilities of $\gamma$-transitions at $\gamma$-ray emission and photoabsorption can be described using the photon (radiative, gamma-ray) strength functions $[1,2]$. These functions are involved in calculations of the observed characteristics of most nuclear reactions. They are also used for investigation of nuclear structure (nuclear deformations, energies and widths of the giant dipole resonances, contribution of velocity-dependent force, shape-transitions, etc.) as well as in studies of nuclear reaction mechanisms. The PSF is important constituent of the compound nucleus model calculations of capture cross sections, $\gamma$-ray production spectra, isomeric state populations, and competition between gamma-ray and particle emission. The most important strength functions in such studies are related to the electric dipole (E1), magnetic dipole and electric quadrupole multipolarities. Different semiphenomenological approaches for the PSF of the E1 transitions were discussed in Refs. [1-7].

In this contribution a new closed-form description of the E1 PSF is presented with allowance for electromagnetic response of the two coupled nuclear states PDR and GDR. In spherical nuclei, the pygmy dipole resonance (PDR) is manifested near the neutron separation energy. It is most pronounced in spherical atomic nuclei with a large neutron excess. Previously in this case the E1 PSF was approximated, as a rule, by two Lorentzianlike curves. They are associated with the response of two independent modes: the PDR mode (oscillations of neutron skin opposite core) and the GDR mode (vibrations in the core of neutrons opposite protons).

Different microscopic and macroscopic approaches indicate an important impact of the relationships between the PDR and GDR modes [7].

We propose a model of two coupled damped state excitations for description of the E1 PSF. The calculations within this approach are compared with those for available experimental data on photoabsorption cross-sections in the

a e-mail: plujko@gmail.com spherical atomic nuclei and microscopic calculations. The input parameters for the calculations are fixed by the use of the experimental data.

\section{PSF with allowance for PDR response}

The photoabsorption cross-section $\sigma_{E 1}$ of dipole electric (E1) field is defined by the photoabsorption photon strength function $\vec{f}_{E 1}$ in the following way:

$$
\sigma_{E 1}\left(E_{\gamma}\right)=3 E_{\gamma}(\pi \hbar c)^{2} \vec{f}_{E 1}\left(E_{\gamma}\right)
$$

where $E_{\gamma}$ is the energy of the gamma-rays. For photoabsorption in cold nuclei, the PSF is determined by the imaginary part of nuclear response function $\chi$ on E1 field

$$
\vec{f}\left(E_{\gamma}\right)=8.674 \cdot 10^{-8} \cdot\left(-\frac{1}{\pi} \cdot \operatorname{Im} \chi\left(E_{\gamma}\right)\right), \mathrm{MeV}^{-3} .
$$

To take into account the relationship between PDR and GDR modes we use nuclear response function of the model of two coupled damped oscillators [8-10] in an external field $E \sim \exp (i \omega t)$ :

$$
\left\{\begin{array}{l}
\ddot{x}_{p}+\left(k_{p}+k\right) x_{p}-k x_{g}+\gamma_{p} \dot{x}_{p}=e_{p} E, \\
\ddot{x}_{g}+\left(k_{g}+k\right) x_{g}-k x_{p}+\gamma_{g} \dot{x}_{g}=e_{g} E,
\end{array}\right.
$$

where $x_{p, g}, \gamma_{p, g}, k_{p, g}$ are displacements, damping widths, restoring forces for nucleons forming pygmy and giant resonances, correspondingly, and the factor $e_{\alpha}(\alpha=p, g)$ determines contribution of the state $\alpha$ to the response function at action of external field.

As it was shown in Ref. [8], the system (3) can be transformed to the equations

$$
\left\{\begin{array}{l}
\ddot{y}_{p}+\omega_{p}^{2} y_{p}+\left(\Gamma_{p}+\gamma\right) \dot{y}_{p}-\gamma \dot{y}_{g}=\mathrm{z}_{p} E \\
\ddot{y}_{g}+\omega_{g}^{2} y_{g}+\left(\Gamma_{g}+\gamma\right) \dot{y}_{g}-\gamma \dot{y}_{p}=\mathrm{z}_{g} E
\end{array},\right.
$$

by the use of the transformation matrix

$$
U=\left(\begin{array}{cc}
\cos \theta, & \sin \theta \\
-\sin \theta, & \cos \theta
\end{array}\right) .
$$


Here, the angle $\theta$ is the solution of the equation:

$$
\cot ^{2} \theta+\frac{k_{g}-k_{p}}{k} \cot \theta=1,
$$

and

$$
\begin{aligned}
& \left(\begin{array}{l}
y_{p} \\
y_{g}
\end{array}\right)=U\left(\begin{array}{l}
x_{p} \\
x_{g}
\end{array}\right), \quad\left(\begin{array}{l}
\mathrm{z}_{p} \\
\mathrm{z}_{g}
\end{array}\right)=U\left(\begin{array}{c}
e_{p} \\
e_{g}
\end{array}\right), \\
& \left(\begin{array}{cc}
\Gamma_{p}+\gamma, & \gamma \\
\bar{\gamma}, & \Gamma_{g}+\gamma
\end{array}\right)=U\left(\begin{array}{ll}
\gamma_{p}, & 0 \\
0, & \gamma_{g}
\end{array}\right) U^{-1},
\end{aligned}
$$

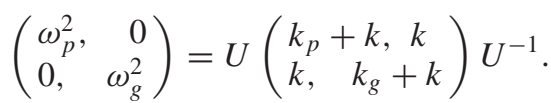

The solution of the system (4) leads to the following analytical expression for the nuclear response function $\chi\left(E_{\gamma}\right)$ on the external field with the frequency $\omega=E_{\gamma} / \hbar$

$$
\chi\left(E_{\gamma}\right)=P\left(E_{\gamma} ; G D R, P D R\right)+P\left(E_{\gamma} ; P D R, G D R\right)
$$

with

$$
\begin{aligned}
& P\left(E_{\gamma} ; k, l\right)= \\
& =\frac{\mathrm{z}_{k}^{2}+\frac{\mathrm{z}_{k} \mathrm{z}_{l} i E_{\gamma} \gamma}{E_{l}^{2}-E_{\gamma}^{2}+i E_{\gamma}\left(\Gamma_{l}+\gamma\right)}}{E_{k}^{2}-E_{\gamma}^{2}+i E_{\gamma}\left(\Gamma_{k}+\gamma\right)+\frac{\gamma^{2} E_{\gamma}^{2}}{E_{l}^{2}-E_{\gamma}^{2}+i E_{\gamma}\left(\Gamma_{l}+\gamma\right)}},
\end{aligned}
$$

where $E_{m}, \Gamma_{m}$ and $z_{m}(m=k, l->P D R, G D R)$ are the energy, width and contribution of the PDR or GDR state; $\gamma$ is the parameter of a coupling between the two excitation modes.

The approach with using expression (2) for the PSF with (5) for the response function is named below as the Two State Excitation (TSE) model in cold nuclei. The TSE expression with zero value of the coupling width $(\gamma=0)$ corresponds to the response of the independent PDR and GDR modes and consists of the two independent Lorentzians.

For hot nuclei, the Modified Lorentzian method [1, 2,12] is used in the TSE model for general expressions between response function and average PSF; that is, the PSF for photoabsorption $(\vec{f})$ and gamma decay $(\overleftarrow{f})$ are calculated by the formulae

$$
\vec{f}\left(E_{\gamma}\right)=F\left(E_{\gamma}, T_{i}\right), \quad \overleftarrow{f}\left(E_{\gamma}\right)=F\left(E_{\gamma}, T_{f}\right),
$$

where

$$
\begin{aligned}
& F\left(E_{\gamma}, T\right)=8.674 \cdot 10^{-8}\left(-\frac{1}{\pi} \cdot \operatorname{Im} \chi\left(E_{\gamma}\right)\right) L\left(E_{\gamma}, T\right), \mathrm{MeV}^{-3} \\
& L\left(E_{\gamma}, T\right)=\frac{1}{1-\exp \left(-E_{\gamma} / T\right)},
\end{aligned}
$$

and $T_{i}$ is the temperature of the initial state in the photoexcitation and $T_{f}$ is the temperature of the final state in gamma-decay; the temperature $T_{i}$ is calculated for the initial excitation energy $E_{i}^{*}$ in photoabsorption and the temperature $T_{f}$ is a function of the final excitation energy $E_{f}^{*}=E_{i}^{*}-E_{\gamma}$ of deexcited nucleus.

The scaling factor $L\left(E_{\gamma}, T\right)$ determines low-energy enhancement of the PSF in heated nucleus and can be interpreted as the average number of the $1 \mathrm{p}-1 \mathrm{~h}$ states excited by an electric field.
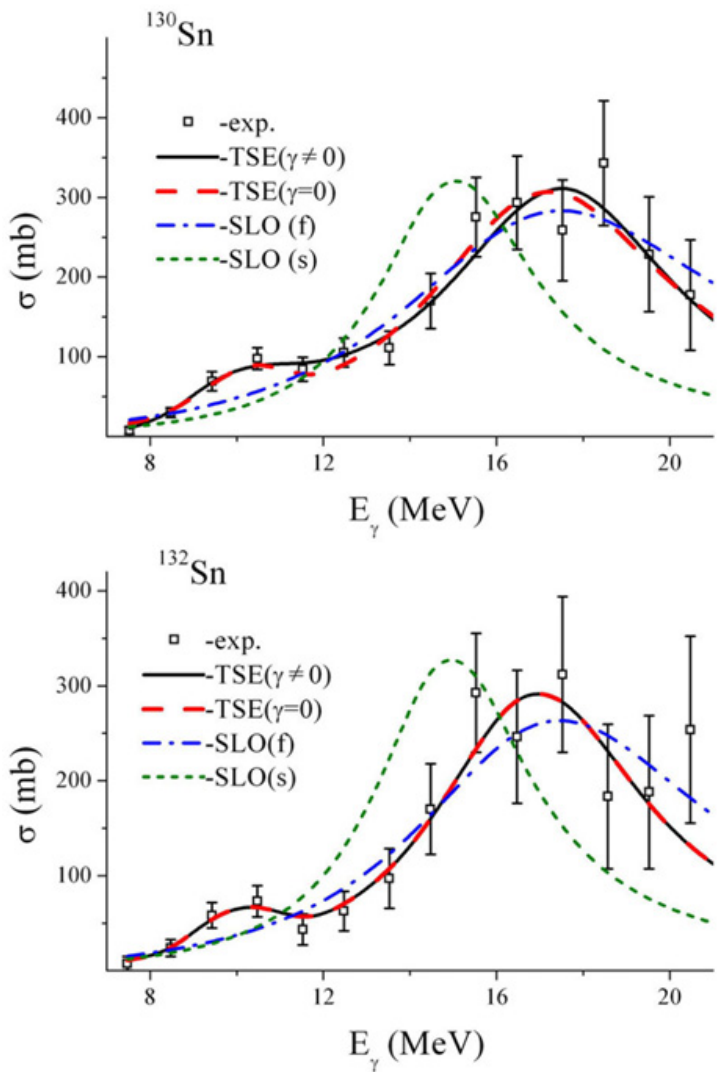

Figure 1. Dependence of the photoabsorption cross-section for ${ }^{130} \mathrm{Sn}$ and ${ }^{132} \mathrm{Sn}$ on gamma-ray energy. The experimental data are taken from [13] and the fitting energy range is $E_{\gamma}<21(\mathrm{MeV})$.

\section{Calculations and discussion}

We apply the TSE model with response of the PDR for description of the different experimental data for photoabsorption cross-sections.

The relationships (1), (2), (5), (6) are used for calculation of the cross-sections. The Figs. 1, 2 show the comparisons of the experimental data with the calculations for the isotopes ${ }^{130,132} \mathrm{Sn}[13],{ }^{88} \mathrm{Sr}$ and ${ }^{139} \mathrm{La}[14,15]$.

The resonance parameters were found by fitting of the experimental data. The initial values of the GDR parameters were taken from the Table 1 of Ref. [4] or from systematics given by Eqs. (2), (3) in Ref. [16].

The SLO-curves (SLO(s), SLO(f)) correspond to calculations within the model of standard Lorentzian in spherical nuclei (one Lorentzian with the constant width): the GDR parameters in SLO(s) were taken from Refs. $[4,16]$ as above; in SLO(f) the parameters were used from fitting experimental data.

The parameters with uncertainties (one-sigma standard deviation) and the least-square deviations $\left(\chi^{2}\right)$ in fitting the calculations to the data are presented in the Tables 1 and 2, where

$$
\chi^{2}=\sum_{j=1}^{j_{\max }}\left(\sigma_{\exp , j}-\sigma_{t h, j}\right)^{2} /\left(\left(j_{\max }-n\right) \cdot \Delta \sigma_{\exp , j}^{2}\right),
$$

$j_{\max }=14-$ the number of the experimental data poins of a cross-section $\left(\sigma_{\exp , j}=\sigma\left(E_{\gamma, j}\right)\right)$ at gamma-ray energy $E_{\gamma, j}<21(\mathrm{MeV}) ; n$-number of the parameters $(n=7$ for TSE with $\gamma \neq 0, n=6$ for TSE with $\gamma=0$ and $n=3$ 

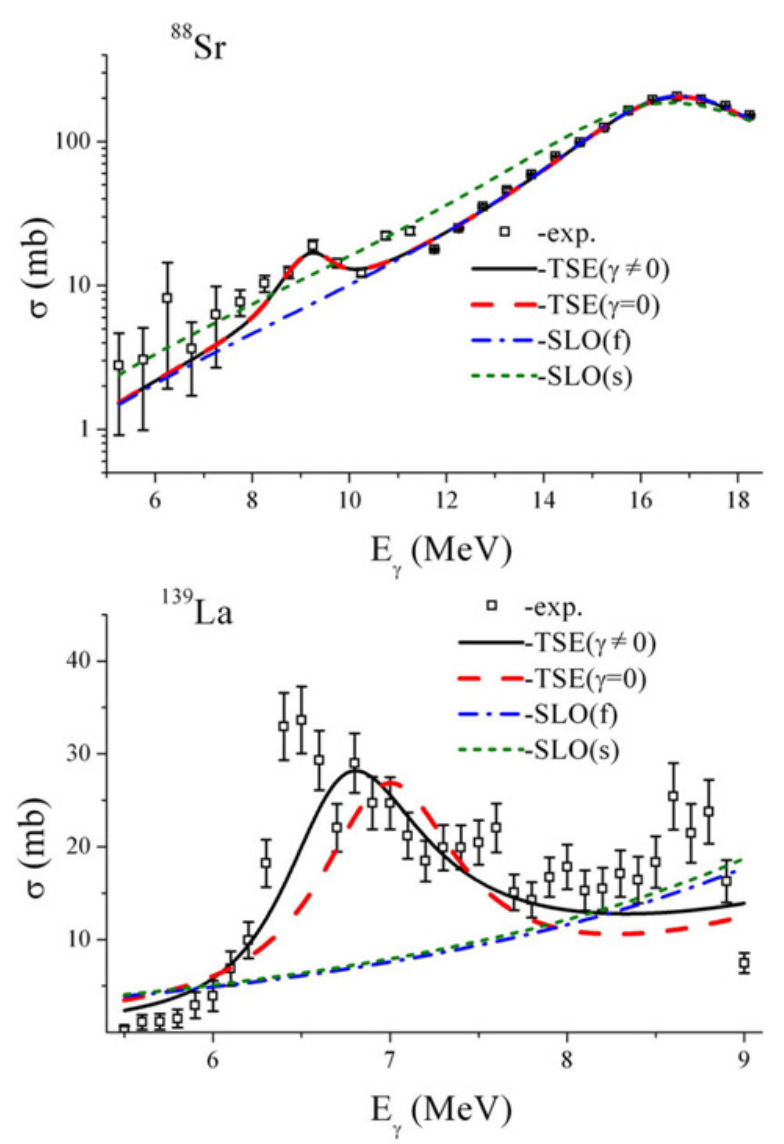

Figure 2. Dependence of the photoabsorption cross-section for the ${ }^{88} \mathrm{Sr}$ and ${ }^{139} \mathrm{La}$ on gamma-ray energy. The experimental data are taken from $[14,15]$.

Table 1. Comparison of the resonance parameters for ${ }^{130} \mathrm{Sn}$.

\begin{tabular}{|c|c|c|c|c|c|}
\hline & TSE $(\gamma \neq 0)$ & TSE $(\gamma=0)$ & SLO(f) & SLO $(s)$ & {$[13]$} \\
\hline$\gamma$ & 2.513 & 0 & - & - & - \\
\hline$E_{P D R}$ & 9.52 & 10.12 & - & - & 10.17 \\
\hline$\Gamma_{P D R}$ & 0.037 & 1.211 & - & - & $<3.4$ \\
\hline$s_{P D R}$ & 0.1184 & 0.094 & - & - & - \\
\hline$E_{G D R}$ & 17.85 & 17.24 & 17.56 & 15.0 & 15.95 \\
\hline$\Gamma_{G D R}$ & 3.615 & 6.612 & 9.459 & 4.5 & 4.817 \\
\hline$s_{G D R}$ & 1.5825 & 1.7612 & 2.2629 & 1.22 & - \\
\hline$\chi^{2}$ & 0.6 & 1.0 & 2.6 & 8.3 & - \\
\hline
\end{tabular}

for SLO). In the tables, the parameter $s_{\alpha}$ is a strength of the corresponding resonance (first or second component in Eq. (5)) in units of the Thomas-Reiche-Kuhn sum rule $\sigma_{T R K}=60 \mathrm{NZ} / \mathrm{A}(\mathrm{mb} \cdot \mathrm{MeV})$ :

$$
s_{\alpha}=\int_{0}^{\infty} \sigma_{\alpha} d E_{\gamma} / \sigma_{T R K}, \quad \alpha=P D R \text { or } G D R .
$$

The PDR parameters obtained from fitting TSE model with $\gamma=0$ to data practically coincide with that ones from Ref. [13]. There are some differences in the values of obtained GDR energies and the results from Ref. [13]. In our opinion, it results from different energy intervals of fitting. For ${ }^{130} \mathrm{Sn}$, the values of the least-square $\chi^{2}$ and GDR width are reduced with allowance for coupling $\gamma$ between PDR and GDR modes. In this case TSE approach with $\gamma \neq 0$ provides better description of the experimental data as compared to the model of the two independent
Table 2. Comparison of the resonance parameters for ${ }^{132} \mathrm{Sn}$.

\begin{tabular}{|c|c|c|c|c|c|}
\hline & TSE $(\gamma \neq 0)$ & TSE $(\gamma=0)$ & SLO $(f)$ & SLO $(s)$ & {$[13]$} \\
\hline$\gamma$ & 0.06 & 0 & - & - & \\
\hline$E_{P D R}$ & 10.01 & 10.01 & - & - & 9.87 \\
\hline$\Gamma_{P D R}$ & 0.528 & 0.528 & - & - & $<2.5$ \\
\hline$s_{P D R}$ & 0.0943 & 0.0925 & - & - & - \\
\hline$E_{G D R}$ & 17.05 & 17.05 & 17.48 & 15.0 & 16.17 \\
\hline$\Gamma_{G D R}$ & 5.513 & 5.513 & 8.117 & 4.4 & 4.721 \\
\hline$s_{G D R}$ & 1.4122 & 1.4126 & 1.8333 & 1.22 & - \\
\hline$\chi^{2}$ & 0.6 & 0.6 & 1.4 & 5.4 & - \\
\hline
\end{tabular}

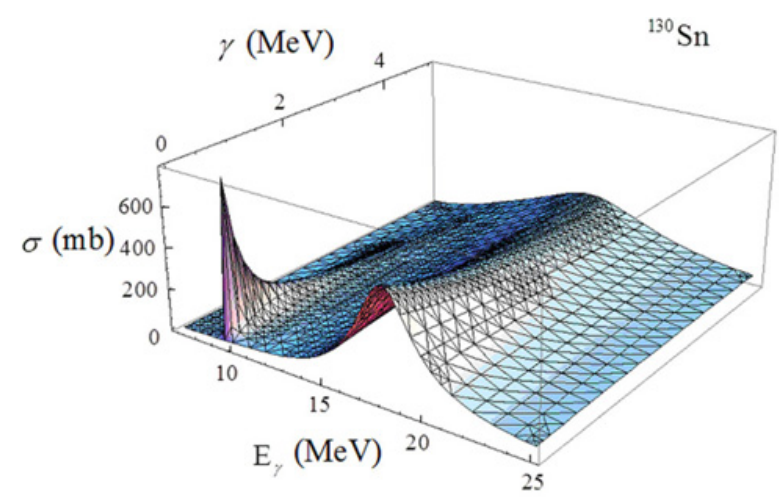

Figure 3. Photoabsorption cross section of ${ }^{130}$ Snversus gammaray energy $E_{\gamma}$ and coupling width $\gamma$. The values of other six parameters were fixed and taken from the first column of the Table 1.

Table 3. The average values $\left\langle\chi^{2}\right\rangle$ of least-square deviations of the theoretical calculations from experimental data for $N$ nuclei.

\begin{tabular}{|c|c|c|c|c|c|}
\hline $\begin{array}{c}\text { Main Ref. } \\
\text { for data }\end{array}$ & $N$ & \multicolumn{4}{|c|}{ Model } \\
\cline { 3 - 6 } & TSE $(\gamma \neq 0)$ & TSE $(\gamma=0)$ & SLO(f) & SLO(s) \\
\hline$[13]$ & 2 & 0.6 & 0.8 & 2.0 & 6.8 \\
\hline$[14,15]$ & 12 & 8.6 & 10.7 & 17.9 & 149.9 \\
\hline$[17]$ & 20 & 8.7 & 13.4 & 14.3 & 224.0 \\
\hline
\end{tabular}

Lorentzians (i.e., TSE with $\gamma=0$ ) and the SLO model and allows for more accurate determination of the GDR parameters. For ${ }^{132} \mathrm{Sn}$, coupling between PDF and GDR mode is not manifested.

Figure 3 demonstrates impact of the coupling parameters $\gamma$ on the shape of the photoabsorption crosssections in ${ }^{130} \mathrm{Sn}$. The GDR and PDR parameters are fixed and listed in Table 1. It can be seen that in the GDR range the peak-value of cross-section is sharply decreased and GDR width is increased with increasing coupling parameter $\gamma$.

Table 3 shows the average values of least-square deviation $\left\langle\chi^{2}\right\rangle=\sum_{i=1}^{N} \chi_{i}^{2} / N$ in fitting the calculations to the data for $N$ nuclei: ${ }^{130,132} \mathrm{Sn}[13],{ }^{88} \mathrm{Sr},{ }^{89} \mathrm{Y},{ }^{90} \mathrm{Zr}$, 92,94,96,98,100 Mo, ${ }^{124,128,134} \mathrm{Xe},{ }^{139} \mathrm{La}[14,15],{ }^{91,92,94,96} \mathrm{Zr}$, 95,97,98, 100 Mo, ${ }^{105,106,108} \mathrm{Pd},{ }^{116,117,118,119,120,122,124} \mathrm{Sn},{ }^{139} \mathrm{La}$, ${ }^{141} \operatorname{Pr}$ [17] (these data were taken from EXFOR database).

It can be seen from the results presented in the Table 3, that TSE approach with nonzero coupling $\gamma$, provides better description of the experimental data in comparison with the model of the two independent Lorentzians (TSE model with $\gamma=0$ ) and SLO model.

Table 4 shows average values $\left\langle\chi^{2}\right\rangle$ of leastsquare deviation of the calculations within different phenomenological models from the microscopic results 
Table 4. The average values $<\chi^{2}>$ of least-square deviations of the phenomenological calculations from microscopic results.

\begin{tabular}{|c|c|c|c|c|c|}
\hline \multirow{2}{*}{ Ref. for data } & \multirow{2}{*}{$N$} & \multicolumn{4}{|c|}{ Model } \\
\cline { 3 - 6 } & & TSE $(\gamma \neq 0)$ & TSE $(\gamma=0)$ & SLO $(f)$ & SLO $(s)$ \\
\hline$[18]$ QRPA & 7 & 7.5 & 15.6 & 26.4 & 2214.6 \\
\hline$[18]$ QTBA & 7 & 8.1 & 20.1 & 34.3 & 653.6 \\
\hline
\end{tabular}

[18] within framework of quasi-particle random phase approximation (QRPA) and the quasi-particle time blocking approximation (QTBA). The microscopic calculations for isotopes ${ }^{58,68,72} \mathrm{Ni},{ }^{116,118,122,132} \mathrm{Sn}$ were used; they were taken from corresponding figures in Ref. $[7,18]$ with energy step $0.01 \mathrm{MeV}$. The uncertainty $\Delta \sigma_{\mathrm{exp}, j}$ for $\chi_{i}^{2}$, Eq. (8), was adopted equal to 10 percent of corresponding microscopic value of $\sigma_{j}$.

It can be seen, that TSE model describes the microscopic calculations much better then SLO approaches.

On the whole, the proposed TSE model is a simple approach to account for E1 strength enhancement in the PDR region. Allowance for coupling between low and high energy modes leads to better description of the experimental data and the microscopic calculations in comparison with situations of the independent modes. Therefore, TSE approach can provide more accurate determination of the resonance parameters (both PDR and GDR).

This work is supported in part by the IAEA (Vienna) under IAEA Research Contract within CRP \#F41032

\section{References}

[1] T. Belgya et al., Handbook for Calculations of Nuclear Reaction Data: RIPL-2, IAEATECDOC-1506 (IAEA, Vienna, 2006); http: //www-nds . iaea.org/RIPL-2/

[2] R. Capote et al., Nucl. Data Sheets 110, 3107 (2009); http: //www-nds . iaea.org/RIPL-3/

[3] A.R. Junghans et al., Phys. Lett. B 670, 200 (2008)

[4] V.A. Plujko et al., At. Data Nucl. Data Tables 97, 567 (2011)

[5] V. Avrigeanu et al., Phys. Rev. C 90, 044612 (2014)

[6] V.A. Plujko et al., Nucl. Data Sheets 118, 237 (2014)

[7] O. Achakovskiy et al., Phys. Rev. C 91, 034620 (2015); https://arxiv.org/abs/1412.0268v1

[8] A.S. Barker, J.J. Hopfield, Phys. Rev. 135, A1732 (1964)

[9] V. Baran et al., Rom. Journ. Phys. 57, 36 (2012)

[10] V. Baran et al., Phys. Rev. C 85, 051601(R) (2012)

[11] A. Croitoru et al., Rom. Journ. Phys. 60, 748 (2015)

[12] V.A. Plujko (Plyuiko), Sov. J. Nucl. Phys. 52, 639 (1990)

[13] P. Adrich et al., Phys. Rev. Lett . 95, 132501 (2005)

[14] A. Makinaga et al., Phys. Rev. C 82, 024314 (2010)

[15] R. Schwengner et al., Phys. Rev. C 78, 064314 (2008)

[16] V.A. Plujko et al., Journ. Korean Phys. Soc. 59(2), 1514 (2011)

[17] H. Utsunomiya et al., Phys. Rev. C82, 064610 (2010)

[18] O. Achakovskiy et al., EPJ Web of Conferences, 107, 05002(2016); EPJ Web of Conferences 107, 05005 (2016) 